\title{
1 Full-field modeling of heat transfer in asteroid regolith 2: Effects of porosity
}

3 Andrew J. Ryan ${ }^{1}$, Daniel Pino-Muñoz ${ }^{2}$, Marc Bernacki ${ }^{2}$, Marco Delbo ${ }^{3}$, Naoya Sakatani ${ }^{4}$, Jens

4 Biele $^{5}$, Joshua P. Emery ${ }^{6}$, Benjamin Rozitis ${ }^{7}$

$6 \quad{ }^{1}$ Lunar and Planetary Laboratory, University of Arizona, Tucson, AZ, USA.

$7 \quad{ }^{2}$ Centre de mise en forme des matériaux (CEMEF), Mines ParisTech, PSL Research University, 8 Sophia Antipolis, France.

$9{ }^{3}$ Observatoire de la Côte d'Azur, CNRS-Lagrange, Université Côte d'Azur, Nice, France.

$10{ }^{4}$ Rikkyo University, Tokyo, Japan.

$11{ }^{5}$ German Aerospace Center (DLR), Köln, Germany.

$12{ }^{6}$ Department of Astronomy and Planetary Sciences, Northern Arizona University, Flagstaff, AZ, 13 USA.

$14{ }^{7}$ School of Physical Sciences, The Open University, Milton Keynes, UK.

15

16 Corresponding author: Andrew Ryan (ajryan4@arizona.edu)

Key Points:

- A 3D finite element model is used to study the effects of regolith porosity and material properties on the radiative thermal conductivity.

- A new, empirical model for regolith radiative thermal conductivity is presented.

- We show that regolith packing density has a minimal effect on predicted regolith particle sizes from thermal inertia on airless bodies. 


\section{Abstract}

The thermal conductivity of granular planetary regolith is strongly dependent on the porosity, or packing density, of the regolith particles. However, existing models for regolith thermal conductivity predict different dependencies on porosity. Here, we use a full-field model of planetary regolith to study the relationship between regolith radiative thermal conductivity, porosity, and the particle non-isothermality. The model approximates regolith as regular and random packings of spherical particles in a 3D finite element mesh framework. Our model results, which are in good agreement with previous numerical and experimental datasets, show that random packings have a consistently higher radiative thermal conductivity than ordered packings. From our random packing results, we present a new empirical model relating regolith thermal conductivity, porosity, temperature, particle size, and the thermal conductivity of individual particles. This model shows that regolith particle size predictions from thermal inertia are largely independent of assumptions of regolith porosity, except for when the nonisothermality effect is large, as is the case when the regolith is particularly coarse and/or is composed of low thermal conductivity material.

\section{Plain language summary}

The temperature of a planetary surface is strongly controlled by the thermal inertia of the surface materials. Specifically, if the surface is covered in a granular regolith, then the size, thermal conductivity, and packing density of the regolith particles strongly affects the surface thermal inertia, which in turn controls surface temperatures. In this work, we use 3D numerical simulations of heat transfer through beds of spherical particles, representing a planetary regolith, to investigate how thermal conductivity and thermal inertia are controlled by the packing density and thermal conductivity of the spheres. Our results are presented in the form of a new empirical model, which could be used to calculate regolith thermal conductivity from knowledge of particle size, composition, and packing density. The use of this model is demonstrated in the typical reverse fashion, where an observed planetary thermal inertia is converted into a predicted regolith particle size. Our model shows that the predicted particle size is largely independent of regolith particle packing density, in contrast to other common regolith models.

\section{Introduction}

The thermal conductivity of planetary regolith can be estimated from remote surface temperature observations using a planetary thermophysical model (e.g., Delbo et al., 2015; Grott et al., 2019; Rozitis et al., 2020). The thermal conductivity of a regolith in vacuum is sensitive to many physical properties of the regolith, such as particle size, porosity (or packing density), and the material properties of the individual particles (e.g., emissivity and thermal conductivity; Wechsler et al., 1972; Kaviany, 1995). Thus, remote estimates of regolith thermal conductivity allow for study of geologic processes that affect the regolith, such as meteoroid bombardment, thermal fracturing, and mass wasting (Cambioni et al., 2021). Robotic and crewed missions furthermore often rely on a constrained knowledge of regolith properties to ensure the success of mission objectives, such as sampling and landing (e.g., Fergason et al., 2006; Emery et al., 2014). 
In this work, we build upon developments from a previous paper (Ryan et al., 2020) to conductivity, porosity, and material properties. We again utilize a $3 \mathrm{D}$ finite element mesh framework where regolith is approximated as ordered and random packings of spherical particles, with the added improvement of periodicity to limit model edge effects. We find a new empirical fit between the radiative exchange factor, used to calculate radiative thermal conductivity, porosity, and regolith particle thermal conductivity and temperature. Our results compare well to experimental datasets and to more limited numerical model results from other researchers. Throughout this work, we use the word "porosity" to denote the relative fraction of void space between regolith particles, otherwise known as macroporosity or the inverse of particle bed packing density. This is not to be confused with microporosity, which is the pore space within an individual regolith particle.

\section{Background}

There are two relevant modes of heat transfer between opaque particles in vacuum radiation between particle surfaces and conduction across the contacts between particles (Wesselink, 1948; Watson, 1964; Wechsler et al., 1972; van Antwerpen et al., 2010). These two heat transfer mechanisms are typically represented in terms of their effective thermal conductivity, where thermal conductivity due to radiative heat transfer is denoted by $\mathrm{k}_{\mathrm{r}}$ and thermal conductivity due to the contacts between the particles, referred to herein as "solid conductivity," is denoted by $\mathrm{k}_{\mathrm{s}}$. The total thermal conductivity of the regolith particulate assemblage may thus be expressed as $\mathrm{k}=\mathrm{k}_{\mathrm{s}}+\mathrm{k}_{\mathrm{r}}$.

In a previous study, we focused our efforts on examining how bulk radiative thermal conductivity, $\mathrm{k}_{\mathrm{r}}$, of a regolith is related to particle size frequency distribution and material properties (Ryan et al., 2020). In this present work, we again focus on investigating the radiative thermal conductivity of regolith for two reasons. First, radiative thermal conductivity on airless bodies is typically much larger than the conductivity due to particle-to-particle contacts in coarse particulate regoliths (i.e., > 5 mm, Ryan et al., 2020, Sakatani et al., 2017; Gundlach and Blum, 2013), which are of high interest for recent missions to rubble-pile asteroids (OSIRIS-REx and Hayabsa2) that have regolith that is likely coarse, where present (Rozitis et al., 2020; Cambioni et al., 2021).

The second reason for focusing on radiative conductivity is that it is much less well constrained as a function of regolith porosity than is conductivity due to contacts; different models predict distinctive trends of $\mathrm{k}_{\mathrm{r}}$ versus porosity. For example, with a doubling in porosity from 0.4 to 0.8, the models by Sakatani et al. (2017) and Gundlach and Blum (2013) predict increases in $\mathrm{k}_{\mathrm{r}}$ that differ by a factor of $\sim 6$. The value of $\mathrm{k}_{\mathrm{s}}$, conversely, is less variable between models and instead depends on the accuracy of the correlation that is used to relate coordination number (i.e., the mean number of contact points per particle) to porosity and assumptions of particle-to-particle cohesion and contact deformation (e.g., Sakatani et al., 2017; Arakawa et al., 2017; 2019). Using the same porosity doubling example, the different coordination number models reviewed in van Antwerpen et al. (2010) predict decreases in $\mathrm{k}_{\mathrm{s}}$ that vary only by a factor of $\sim 2$ between. 


\subsection{Radiative thermal conductivity vs. regolith porosity}

The thrust of this work is to determine the relationship between $\mathrm{k}_{\mathrm{r}}$ and porosity (or interparticle void fraction) of a particulate regolith. As previously mentioned, different models use different theoretical frameworks to approximate this relationship and thus lead to appreciably different results, particularly for regoliths with high macroporosity that might be found in microgravity environments such as small bodies (Murdoch et al., 2015). It is useful to summarize here the general approximation that is used as the starting point for radiative heat transfer in sphere beds - layers of spheres are approximated as a series of parallel plates (e.g., Wesselink, 1948). The general formulation is:

$(\mathrm{Eq} 1)$

$$
k_{r}=4 \sigma F d_{p} \bar{T}^{3}
$$

where $\sigma$ is the Stefan-Boltzmann constant, $F$ is a radiative exchange factor, $d_{p}$ is the sum of the plate half thickness and the gap half thickness (later this will be particle diameter when we use this to describe sphere beds), and $\bar{T}$ is the mean temperature (Wesselink, 1948; Jakob, 1957). In the true case of heat transfer by radiation across a series of parallel plates, $F$ is simply a function of hemispherical emissivity $(\varepsilon)$ of the plates, i.e., $F=\varepsilon /(2-\varepsilon)$.

Approximating a packing of regolith particles as a series of perfectly opaque layers is obviously a huge oversimplification. The radiative exchange factor, F, serves to bridge the gap between this approximation and the bed of particles that constitutes a regolith. Many have sought to define the radiation exchange factor or sought other novel methods to approximate or directly model heat transfer in packed beds of spheres, especially in literature related to pebble bed nuclear reactors (van Antwerpen et al., 2010; de Beer et al., 2018; Calderón-Vásquez et al., 2021) and other industrial applications (Vortmeyer, 1979; Tausendschön and Radl, 2021). However, many of these studies have only considered packings across a narrow range of porosity values (e.g., $0.4-0.5$ ), which are in general too narrow for planetary science applications. Upon finding a small sensitivity in $F$ to porosity within this range, some concluded that porosity was not worth consideration compared to other factors that tend to vary more widely in industrial applications, such as the emissivity (e.g., Singh and Kaviany, 1994).

Recent regolith thermal conductivity models have suggested that $F$ could be quite sensitive to porosity across the full range of regolith microporosities relevant to planetary regolith, yet they differ considerably in their predictions. Sakatani et al. (2017) assume that $F$ is chiefly related to the length of the void spaces present between particles. The voids are approximated as having a spherical shape; $F$ is used to relate particle diameters in Equation 1 to porosity:

$(\mathrm{Eq} 2)$

$$
F=\frac{\varepsilon}{2-\varepsilon} \zeta\left(\frac{\phi}{1-\phi}\right)^{1 / 3}
$$


where $\phi$ is the regolith porosity and $\zeta$ is an empirical correction coefficient obtained from experimental data. Laboratory measurements of the bulk thermal conductivity of glass beads (Sakatani et al., 2017) and basaltic particles (Sakatani et al., 2018) indicated that $\zeta$ may have a particle size dependence. The exact physical cause of this is not clear, however it may be due to a breakdown in the assumption that each particle is an independent scatterer of light as particle size approaches the dominant thermal infrared wavelengths (Wada et al., 2018).

Glundlach and Blum $(2012 ; 2013)$, to the contrary, rely on the assumption that $\mathrm{F}$ is controlled by the mean free path of the photon:

(Eq 3)

$$
F=\varepsilon e_{1} \frac{\phi}{1-\phi} *\left[\frac{2}{3}\right]
$$

where $e_{1}$ is an empirical constant, the value of which was estimated to be $\sim 1.33$ or $\sim 4 / 3$ based on simulations of gas particle diffusion through porous media by Skorov et al. (2011).

The value of 2/3 in brackets is used in Gundlach and Blum (2012) based on a formulation for $F$ from Merrill (1969). However, in a follow-up work (Gundlach and Blum, 2013) the authors omit this additional factor of $2 / 3$ in favor of a formulation of $F$ referenced to Schotte (1960). Interestingly, $e_{1} \frac{\phi}{1-\phi}$ is nearly identical to the equation for the hydraulic diameter of a pore in a porous medium. A recent model by Wood (2020) uses a similar formulation and more clearly ascribes it to the Kozeny-Carman law for viscous fluid flow in a porous medium.

Another noteworthy model for radiative thermal conductivity in a sphere bed was presented by van Antwerpen et al. (2012). Radiative conductivity is broken into two terms to describe heat transfer between directly adjacent spheres ("short-range") and radiation between non-adjacent spheres ("long-range"). They also include a sphere non-isothermality correction expression, based on the formulation introduced by Singh and Kaviany (1994) that we will discuss in the next section. The formulations for $F$ for short-range radiative heat transfer is a function of the number of surrounding spheres (i.e., average coordination number), the view factor between touching spheres, the average contact angle (i.e., the average angle between the net heat flow vector and the vector connecting two spheres), and the emissivity of the spheres. For long-range radiation, $F$ depends on the decay in average sphere-to-sphere view factor with distance. They use an average sphere distance and an average view factor, based on a plot of view factor versus distance, and an empirical correction factor, to the calculation of long-range $F$. The decay in view factor with distance would depend on the packing density of the sphere bed. Given that this model was tailored to describe pebble bed nuclear reactors, the porosity is set to approximately 0.39 . In order to apply their model to our work, we would need to find a new expression for the view factor decay with distance as a function of sphere bed porosity, which is challenging. As such, we do not use their model directly but will refer later to the concept of long-range and short-range radiation in the discussion of our results.

Finally, a recent formulation for $F$ was obtained from a numerical view-factor matrix model (Wu et al., 2020). The study specifically focuses on the effect of porosity: 
188

189

190

191

192

193

194

195

196

197

198

199

200

201

202

203

204

205

206

207

208

209

210

211

212

213

214

215

216

217

218

219

220

221

222

223

224

225

226

(Eq 4)

$$
F=\varepsilon\left[a+b\left(\frac{\phi}{1-\phi}\right)^{c}\right]
$$

where empirical constants $a=0.8049, \mathrm{~b}=0.3728$, and c $=1.6214$ produce an excellent fit to their numerical results for porosity values in the range of $\sim 0.26-0.51$.

2.2 Radiative thermal conductivity and the non-isothermality effect

The simplified form of radiative thermal conductivity of a series of parallel plates in Equation 1 relies on the following approximation:

$(\mathrm{Eq} 5)$

$$
\frac{\left(T_{a}^{4}-T_{b}^{4}\right)}{\left(T_{a}-T_{b}\right)} \approx 4 \bar{T}^{3}
$$

where $T_{a}$ and $T_{b}$ are the temperatures of two adjacent plates and $\bar{T}$ is the mean temperature (Wesselink, 1948). This approximation is valid if two assumptions are true: the temperature difference between the two plates is much smaller than the mean temperature, and the temperature gradients within each plate are much smaller than the temperature difference between two adjacent plates (i.e., each plate is approximately isothermal). The first assumption is almost universally valid in planetary regoliths, as exhibited by a simple example: If $T_{a}=300 \mathrm{~K}$ and $T_{b}=350 \mathrm{~K}$, which is likely a much larger temperature gradient than would ever be found between two adjacent regolith particles, the two sides of Equation 5 differ only by an error of $\sim 0.6 \%$. Thus, this assumption would almost universally be valid in cases of planetary regolith, even under extreme cases, such as in the uppermost particle layers of the lunar regolith (e.g., Henderson and Jakosky, 1994).

The second assumption that the plates or the particles are essentially isothermal was recently found to be violated in some planetary regolith cases (Ryan et al. 2020) and has been described for sphere beds in industrial applications by several others (Breitbach and Barthels, 1980; Robold, 1982; Singh and Kaviany, 1994; van Antwerpen et al., 2012). The magnitude of a temperature gradient across a plate or particle, compared to the overall gradient across the series, is related to the thickness of the plates and to their thermal conductivity. This assumption of plate isothermality is generally valid when this approximation is applied to planetary regoliths because most regolith particles on commonly studied bodies like the Moon and Mars are small (sand or smaller) and are made out of geologic materials with relatively high thermal conductivity values. However, Ryan et al. (2020) showed that regolith particles on rubble-pile asteroids like Bennu and Ryugu could have significant thermal gradients due to their large size ( $\sim \mathrm{cm}$ scale) and apparently low thermal conductivity (e.g., Rozitis et al., 2020; Shimaki et al., 2020; Cambioni et al., 2021). This so-called non-isothermality effect acts to reduce the temperature-dependence of the bulk radiative thermal conductivity. That is, equation 1 no longer follows $\mathrm{T}^{3}$ and instead relies on the inclusion of a non-isothermal correction factor, $f_{k}$. The non-isothermality effect was parameterized by Singh and Kaviany (1994) and van Antwerpen et al. (2012) as a function of a dimensionless parameter, $\Lambda_{\mathrm{s}}$ : 
$228(\mathrm{Eq} 6)$

$$
\Lambda_{s}=\frac{k_{m}}{4 D \sigma T^{3}}
$$

where D is the particle diameter (or, the Sauter mean particle diameter in the case of polydisperse packings, Ryan et al. 2020). The non-isothermal correction factor, $f_{k}$ is then calculated as:

$$
f_{k}=a_{1} \tan ^{-1}\left(a_{2}\left(\frac{1}{\Lambda_{s}}\right)^{a_{3}}\right)+a_{4}
$$

where $a_{1}, a_{2}, a_{3}$, and $a_{4}$ are empirical constants. Ryan et al. (2020) calculated new values for these constants using finite element method (FEM) simulations of heat transfer through dense random packings of monodisperse and polydisperse spheres with porosities spanning a relatively narrow range $(\sim 0.35-0.39)$.

Our equation for radiative conductivity now reads:

$243(\mathrm{Eq} 8)$

$$
k_{r}=4 \sigma F(\varepsilon, \phi) f_{k}\left(k_{m}(T), D, T\right) d_{p} \bar{T}^{3}
$$

\section{Methods}

\subsection{Finite Element Model}

We use the FEM to model heat transfer in regolith that is approximated as a 3D meshed geometry of spheres, where each sphere represents a regolith particle. A constant heat flux is applied to a plate on one side of a three-dimensional, parallelepiped-shaped geometry of packed spheres while a constant temperature boundary condition is applied to a plate on the opposite side (Figure 1). Once a steady state temperature distribution is achieved, the bulk thermal conductivity of the system can be calculated from the temperature difference between the two plates, the distance between the plates, and the applied heat flux. These methods are described in more detail in Ryan et al. (2020). As in that work, bulk radiative thermal conductivity is studied exclusively here by removing the contacts between spheres. Nevertheless, heat diffusion within any individual sphere is still modeled and is responsible for the non-isothermality effect described later.

One key improvement that has been implemented since our previous work is the addition of periodicity. All sphere packings in this study are periodic in the two spatial directions ( $\mathrm{x}$ and $y)$ that are orthogonal to the direction of heat flow (z), so as to create the illusion that the 
geometry is infinitely wide geometry, which acts to minimize boundary effects. Surface-tosurface radiative heat transfer is then made periodic by modifying the ray tracing step that is performed to determine which surface mesh elements are visible to each other for heat transfer. During this step, the surface mesh geometry is temporarily duplicated and translated to the eight possible locations immediately surrounding the original geometry (i.e., $+\mathrm{x},+\mathrm{x}+\mathrm{y},+\mathrm{y},+\mathrm{x}-\mathrm{y}$, etc.) in order to create one layer of heat transfer periodicity.

All simulations were conducted with monodisperse sphere packing geometries with sphere diameters $\leq 1 \mathrm{~cm}$. Our model is not currently able to handle non-unitary emissivity, so in all simulations the emissivity is unitary. Additionally, surfaces are assumed to have a Lambertian thermal emission phase function. The thermal conductivity of the sphere material $\left(k_{m}\right)$ was varied between 0.025 and $30 \mathrm{~W} \mathrm{~m}^{-1} \mathrm{~K}^{-1}$. Prescribed heat flux values were chosen based on the thickness and bulk thermal conductivity of each sphere bed in order to minimize thermal gradients across the entire bed to $<10 \mathrm{~K}$. Flux values were in the range of $\sim 2.5-10 \mathrm{~W} \mathrm{~m}^{2}$. Sphere bed thicknesses were within the range of $\sim 4-14 \mathrm{~cm}$. Thicker beds were necessary for higher porosity packings in order to better capture to minimize edge effects caused by the boundary plates. In order to determine optimal geometry thicknesses, we performed a series of tests of varying thickness with different packing types and different packing density (porosity) values. The results of this are described in the supplemental materials.
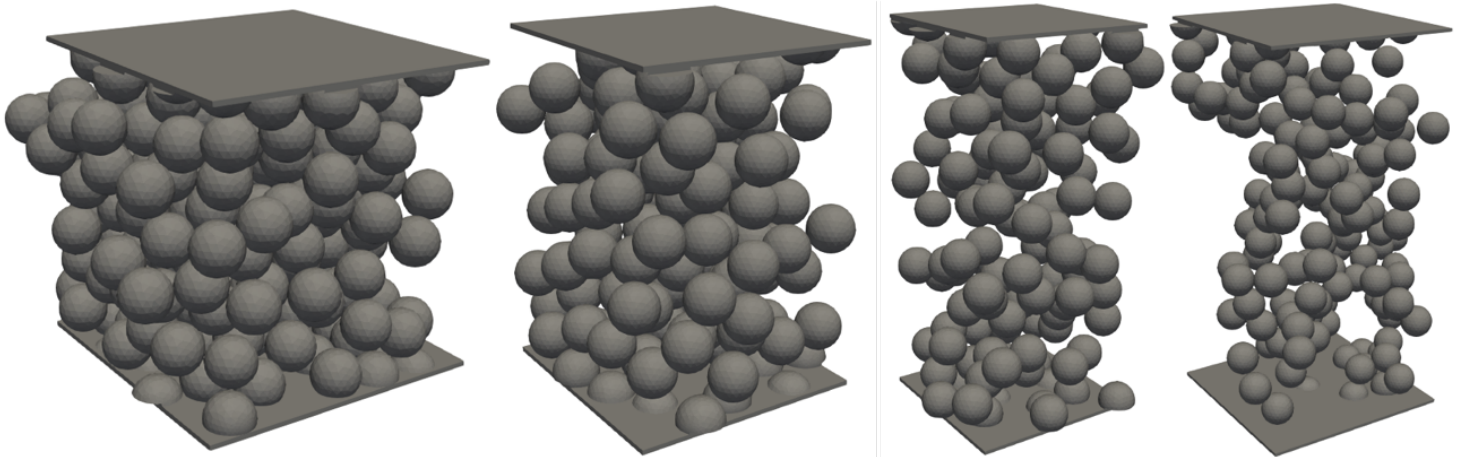

Figure 1. Random packing examples used in this work. The average porosity values are (left to right) $0.47,0.60,0.71$, and 0.81 . All packings are periodic in the lateral directions. The packing methods used (left to right) are Optimized Dropping and Rolling, Ballistic Deposition (single sphere), Random Sequential Packing, and Ballistic Deposition (four-sphere clusters).

We utilized an improved method for extracting bulk thermal conductivity from the numerical simulation results. In the previous work (Ryan et al., 2020), the bulk thermal conductivity was determined from the steady-state temperature difference and distance between the two end plates and the prescribed heat flux using Fourier's law. In this work, we found that the porosity within a given sphere packing could in some cases be highly variable and thus decided to instead calculate local conductivity in discrete slices of each geometry. To do so, the output steady-state temperature solution mesh is divided into $1 \mathrm{~cm}$ thick layers (equivalent to 1 sphere diameter in most simulations). The average temperature of the top and bottom plane of each slide is calculated. The difference between the temperatures of these two planes provides us 
298

299

300

301

302

303

304

305

306

307

308

309

310

311

312

313

314

315

316

317

318

our $\Delta T$. With this, the known prescribed heat flux $(q)$, and the known slide thickness $(\Delta x)$, the local bulk thermal conductivity for each slice is calculated with Fourier's law:

(Eq 9)

$$
k_{r}=q \frac{\Delta x}{\Delta T}
$$

Bulk thermal conductivity is then converted to the non-dimensional radiative exchange factor using:

$(\mathrm{Eq} 10)$

$$
F=\frac{k_{r}}{4 \sigma d_{p} \bar{T}^{3}}
$$

where $\bar{T}$ is the mean temperature within a given slice.

The local porosity is also calculated for each individual slice. The final results are presented as the mean values of $\mathrm{F}$ versus porosity from all slices within a sphere packing geometry, excluding a few (1-3) slices nearest to the boundary plates (depending on geometry thickness) where it was found that $\mathrm{F}$ was consistently lower than in the central region due to edge effects (Figure S1). Error bars in F vs porosity space are the maximum and minimum respective values found among the slices within a given geometry, again excluding slices suspected to be affected by edge effects.

The value of the non-isothermality correction factor, $f_{k}$, is calculated like in Ryan et al. (2020) by comparing pairs of thermal simulation results - one where the non-isothermal effect is negligible and another where it is expected to be significant ( $\gtrsim 1 \%)$. Ryan et al. (2020) assumed that the non-isothermal effect is only significant when $1 / \Lambda_{s}>\sim 0.04$, based on previous work by van Antwerpen et al. (2012). Our approach in this work is more conservative, such that we use simulation results for $F$ where $1 / \Lambda_{\mathrm{s}} \leq 0.0035$ as our baseline values against which we determine the non-isothermal correction factor. In these baseline cases with negligible intraparticle non-isothermality, we use a material thermal conductivity of $k_{m}=30 \mathrm{~W} \mathrm{~m}^{-1} \mathrm{~K}^{-1}$. Subsequent simulations are then performed with lower values of $k_{m}$, which increases $1 / \Lambda_{\mathrm{s}}$ and creates non-isothermality within particles. The value of $f_{k}$ is then calculated by comparing the resulting value of $F$ where $k_{m}<30 \mathrm{~W} \mathrm{~m}^{-1} \mathrm{~K}^{-1}$ to the previously determined baseline value of $F$ where $k_{m}=30 \mathrm{~W} \mathrm{~m}^{-1} \mathrm{~K}^{-1}$ :

\section{$(\mathrm{Eq} 11)$}

$$
f_{k}=\frac{F_{k_{m}<30}}{F_{k_{m}=30}}
$$


Uncertainty in $f_{k}$ is calculated using the same values used for the error bars in $\mathrm{F}$, that is $f_{k, \text { maxerror }}=\left(F_{k_{m}<30, \max } / F_{k_{m}=30, \min }\right)$ and respectively for the minimum error value.

\subsection{Sphere packing methods}

In order to determine if the details of a random packing are influential on the bulk radiative conductivity, we utilized several methods to generate the random sphere packings with different porosity values (Figure 1). The Ballistic Deposition method begins with a seed sphere or a simple seed cluster of spheres in the periodic domain space. New spheres or sphere clusters are then brought from a random location outside of the domain and following a random trajectory. If the sphere or cluster touches an existing sphere within the domain, it sticks immediately. The new addition is kept as long as it does not violate periodicity. This process is repeated many times until the cluster has grown to fill the periodic domain space so that any new spheres or clusters that are brought in are rejected, even after a very large number of attempts $\left(\sim 10^{5}\right)$. Different porosity values may be achieved depending on if individual spheres or sphere clusters are used in the deposition. For example, single sphere deposition can be used to generate packings with porosities in the range of $\sim 0.59-0.61$. Deposition by clusters that contain 2 spheres leads to porosities of $\sim 0.67$, whereas 3 -sphere clusters lead to $\sim 0.72$ and 4 -sphere clusters lead to $\sim 0.74$ and higher.

The Random Sequential Packing method quite simply involves the introduction of a new sphere in the 3D periodic domain space in some random location. If the sphere does not overlap an existing sphere and does not violate periodicity, it is kept. The spheres are not touching each other in this method, so it is not as representative of a natural regolith. However, it has the flexibility of a wide range of achievable porosity values. The densest possible packing that we have achieved with this method has a porosity of $\sim 0.63$.

The Optimized Dropping and Rolling is the same as that described by Hitti and Bernacki (2013) but modified to add periodicity. Spheres are dropped into the periodic domain space from above and roll into a stable position in order to achieve a loose random packing (porosity $\sim 0.43-$ $0.45)$.

Finally, the method by Ringl et al. (2012) is used, where spheres are sequentially attached to pre-existing spheres in spaces in the geometry with low local packing fraction values (see also Ballouz et al., 2021). The method can be terminated once a desired packing density is reached, or allowed to run until no further sphere sites can be found after a large number of attempts, as with the Random Sequential Packing Method. We achieved packings with porosity values as low as $\sim 0.57$ with this method.

Ordered cubic packing structures (a.k.a. "regular" or "structured" packings) were also tested in order to examine low-porosity packings. Simple cubic, body-centered cubic, and facecentered cubic packings have porosity values of $\sim 0.48, \sim 0.32$, and $\sim 0.26$, respectively. The diameters of the spheres in these three packings were then reduced in order to achieve higher porosity values while maintaining an ordered arrangement, although the spheres in those configurations are no longer touching and thus the packings are not possible in nature. 


\subsection{Method for investigating particle roughness}

In addition to the measurements of monodisperse particles described above, a limited set of simulations were conducted with non-spherical particles as preliminary assessment of the effects of particle angularity. Random packings of spheres were generated using the methods described above. The spheres were then "roughened" by the addition of craters to the sphere surfaces. The craters were placed on each sphere by randomly choosing a surface coordinate on the sphere for the placement of another small sphere that served as a subtractive object. In order to qualitatively maximize the roughness induced by these craters on the host spheres while maintaining some semblance of the original spherical particle shape, these spherical section craters were constrained to have radii between $1 / 4$ and 2/3 the diameter of the host sphere. Finally, the spherical section craters were restricted to be placed within pi/3 radians of the poles of the host spheres pointing in the direction of heat flow, in an attempt to maximize the effect of the roughness on heat transfer in a manner similar to asteroid thermal models where spherical section craters are used to approximate topographic roughness (e.g., Spencer, 1990; Rozitis and Green, 2011).

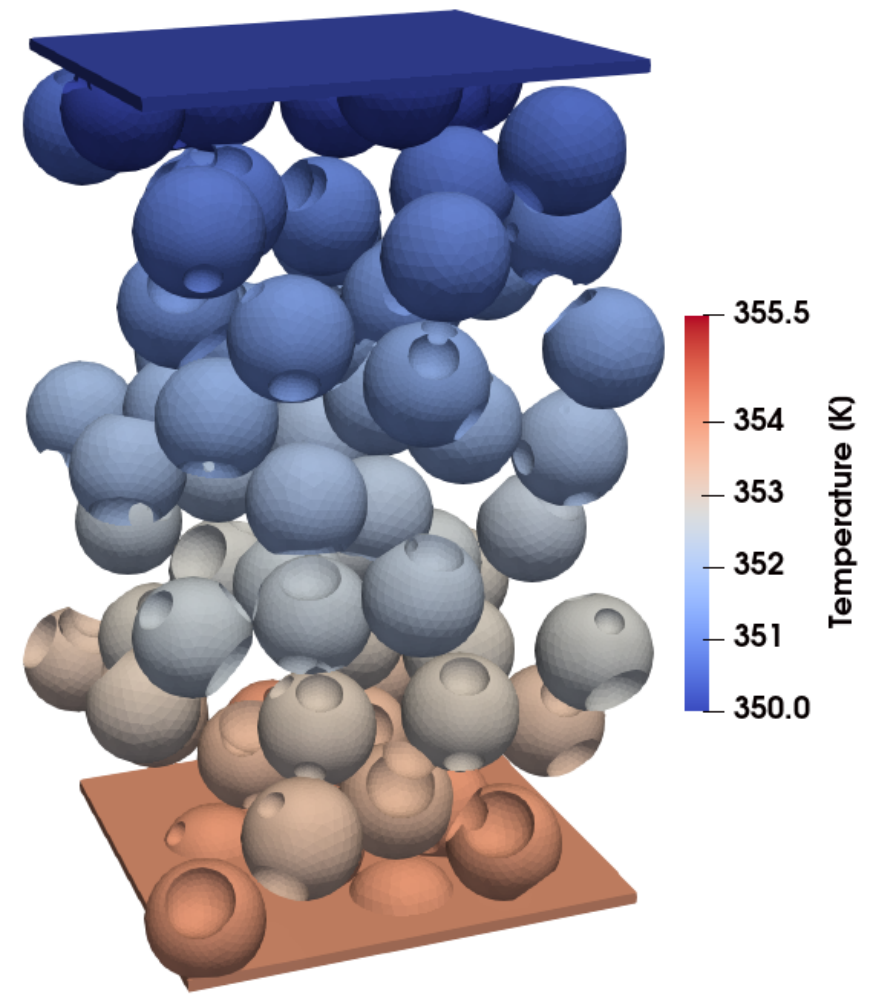

393 Figure 2. Particles with surfaces roughened by the addition of spherical section craters. The colors represent the final, steady-state temperatures. 


\subsection{Preparation of experimental data for comparison}

The recent experimental datasets by Sakatani et al., 2017 and 2018 are ideal for comparing to our model results, given that they measured somewhat coarse particles (up to $\sim 1$ $\mathrm{mm}$ diameter), where radiative thermal conductivity is significant, using a well-established method (line heat source, e.g., Presley and Christensen, 1997). Measurements of bulk thermal conductivity are made with glass spheres and with the JSC1A lunar regolith simulant in different size fractions and at different temperatures, in the range of $\sim 250-330 \mathrm{~K}$. The relative contributions of the radiative and solid conductivity terms are determined by fitting their regolith thermal conductivity model to the temperature dependent results, where the radiative conductivity term is assumed to be proportional to $\mathrm{T}^{3}$ and the solid conductivity term is assumed to be proportional to the conductivity of the particulate material, which is typically only minimally temperature-dependent (e.g., Opeil et al., 2020). Sakatani et al., 2017 and 2018 express their results in terms of tunable parameters for solid conductivity $(\xi, x i)$ and radiative conductivity ( $\zeta$, zeta, equation 2 ). The model of Sakatani et al., 2017 is in good agreement with our numerical results for porosities $\lesssim 0.60$ when $\zeta$ is $\sim 1.25-1.4$ and emissivity is unitary. Their experimental results for the two largest particle ranges tested (355-500 and 710-1000 $\mu \mathrm{m})$ show that $\zeta$ is consistently lower in the JSC1A samples compared to the glass beads (Sakatani et al., 2018, see Fig. 13b within). The absolute values of radiative conductivity and F show that their measurements are significantly lower than our model results. Below, we re-evaluate the way that radiative conductivity was calculated from their experimental data in an effort to reassess how well their measurements agree with our results.

In order to calculate radiative thermal conductivity and radiative exchange factor from the experimental measurements of bulk conductivity by Sakatani et al. (2018) for comparison to our work, one must have some knowledge of the sample particle size, porosity, emissivity, and the thermal conductivity of the individual sample particles. We will revisit the values chosen for each of these parameters.

First, a single, representative particle size must be assumed for each experimental sample. The two coarsest samples tested in JSC1-A had particle sizes of 355-500 and 710-1000 $\mu \mathrm{m}$. These size bins are relatively large; they each span $\sim 40 \%$ in particle size. Sakatani et al. (2018) used the average of the two bounding values for each size range in their data interpretation calculations ( $427 \mu \mathrm{m}$ and $855 \mu \mathrm{m}$, respectively). Conversely, the bulk thermal conductivity of the entire JSC1-A regolith (unsorted) was found to be well-represented by the volumetric median particle size. This result was assumed to be valid for both the solid and radiative thermal conductivity terms; the experimental data set is not comprehensive enough to constrain the effective particle size of the two thermal conductivity components separately. Alternatively, Ryan et al. (2020) found that the Sauter mean particle size is representative of the bulk for the purpose of calculating effective radiative thermal conductivity. In many cases, the Sauter mean and the volumetric median particle size are very similar, including in the sphere packings studied by Ryan et al. (2020). We calculate both for the two aforementioned sample size fractions, assuming that the distribution within each size fraction is the same as in the bulk sample, parameterized using log-normal distribution by mass with distribution parameters $\mu=4.66$ and $\sigma=0.972$ in units of $\ln (\mu m)$ (Sakatani et al., 2018). In both cases, the volumetric median and Sauter mean particle sizes are within a few percent of each other. We arbitrarily choose the volumetric median as the new effective particle size for the two samples in our calculations of radiative thermal conductivity and radiative exchange factor. As such, the effective particle size 
444 of the $355-500 \mu \mathrm{m}$ sample is $412 \mu \mathrm{m}$ rather than the original range-based mean value of $427 \mu \mathrm{m}$. The $710-1000 \mu \mathrm{m}$ value is $816 \mu \mathrm{m}$ rather than $855 \mu \mathrm{m}$.

The porosity of each sample was determined by Sakatani et al. (2018) from the measured bulk density (from measured sample mass and sample container volume) and an assumed particle density (a.k.a. specific gravity) value of $2900 \mathrm{~kg} \mathrm{~m}^{-3}$, which is an average measured value for JSC1A (McKay et al., 1994; Zeng et al., 2010). However, the measured bulk density and porosity values vary substantially between sample size fractions, from $1540 \mathrm{~kg} \mathrm{~m}^{-3}$ and porosity of 0.47 in the smallest size fraction $(53-63 \mu \mathrm{m})$ to $980 \mathrm{~kg} \mathrm{~m}^{-3}$ and porosity of 0.66 in the largest size fraction $(710-1000 \mu \mathrm{m})$. All samples were loaded into the sample container using the same methods; each sample was poured into the container and then tapped. Thus, it is not readily apparent why the packing density values should differ so significantly. Rather, we suspect that the assumption of a constant particle density between the different size fractions is responsible for the different measured bulk density values.

The JSC1-A lunar regolith simulant, described as a volcanic ash of basaltic composition (McKay et al., 1994), was produced by crushing and impact milling basalt cinders or "basaltic welded tuff' (Taylor et al., 2005) from a cinder ash quarry on the flank of Merriam Crater cinder cone in the San Francisco Volcanic Field (Sibille et al., 2006); in particular, it was selected for its high glass content $(\sim 50 \%)$. Scanning electron micrograph images of smaller size fractions $(<$ few hundred $\mu \mathrm{m}$ ) show that the "glassy particles invariably display broken vesicles with sharp edges" (McKay et al., 1994). The larger particles $(\sim 500-1000 \mu \mathrm{m})$ have abundant vesicles that are visible by eye or hand lens. As such, it may be expected that vesicularity, or porosity, should vary between size fractions, where the larger particles are more likely to contain complete vesicles in their interiors and edges. Tamari et al. (2005) for example found that particle density of a scoria varied as a function of particle sizes in the range of $\leq 4.75 \mathrm{~mm}$ to $<74 \mu \mathrm{m}$. Conversely, Zeng et al. (2010) measured the specific gravity of JSC1A in two size fractions, separated by the $75 \mu \mathrm{m}$ sieve, and found no difference. Both of these studies utilized a water pycnometry method, in which water is likely to penetrate into some of the vesicles, excluding them from the particle density analysis and thus leading to density overestimates. We suspect that the fraction of JSC1A larger than $75 \mu \mathrm{m}$ measured by Zeng et al. (2010) still contained abundant crushed, fine particles that did not contain closed vesicles and thus the measurement result was insensitive to any larger particles that did contain closed vesicles. Following the particle size weighted mass distribution for JSC1-A in Sakatani et al. (2018), the sample that is $>75 \mu \mathrm{m}$ tested by Zeng et al. (2010) would have consisted of $80 \%$ by mass particles smaller than $300 \mu \mathrm{m}$.

No other information on the particle density of the coarser particles in JSC1-A could be found in the literature. Direct measurement of the particle density should be conducted with a method that takes all vesicles into account, including those that may not be penetrated by water (e.g., Garboczi, 2011). Nonetheless, we performed a new water pycnometry measurement of the four size fractions used in Sakatani et al. (2018). A small but significant density decrease is noted in the larger size fractions. However, we suspect that these values still over-estimate the true density, given that water certainly filled pores along the edge of the sample and may have also penetrated interior pores, depending on the degree of pore connectivity. For example, if we assume that all samples have the same porosity of 0.45 , which would be a very loose random packing, the microporosity values of the two largest size fractions would be approximately 0.30 and 0.39 (Table S4 and S5). As such, we will re-evaluate the Sakatani et al. 2018 results with these newly measured density values but will also consider the possibility that the microporosity 
489

in the larger size fractions could still be higher, such as would be the case if all samples actually had the same or similar porosity values but different microporosity values.

The assumed material thermal conductivity in Sakatani et al. (2018) comes from experimental measurements of a non-porous basalt and displays an inverse relationship between conductivity and temperature that is common in mineral-rich samples. However, as described above, the JSC1-A simulant is a mixture of minerals and volcanic glass. Although thermal conductivity values of felsic volcanic glasses (e.g., obsidian) can be found in the literature, we were only able to find one instance of a mafic glass measurement (Birch and Clark, 1940). The sample, as described by Birch and Law (1935) was a diabase that was melted in the laboratory and cooled to form a glass that in thin section was "quite free from crystallites... and almost entirely free from gas vesicles". The major element concentrations are similar to those reported for the JSC1 simulant (McKay et al., 1994). The two have a similar theoretical room-temperature glass thermal conductivity calculated from their composition using the glass phonon thermal conductivity model of Choudhary and Potter (2005) $\left(\sim 1.19 \mathrm{~W} \mathrm{~m}^{-1} \mathrm{~K}^{-1}\right.$ for the diabase glass vs. $\sim 1.14 \mathrm{~W} \mathrm{~m}^{-1} \mathrm{~K}^{-1}$ for the JSC1A). This similarity indicates that the diabase glass is a sufficient compositional match to serve as a thermal conductivity analog for the glass component of the JSC1-A. We performed a linear least-squares fit to the thermal conductivity data for the diabase glass provided from $0-300^{\circ} \mathrm{C}$ in Birch and Clark (1940), obtaining $\mathrm{k}_{\text {glass }}=0.846+1.11 \mathrm{e}-3 * \mathrm{~T}$ where $\mathrm{T}$ is temperature in Kelvin. We ultimately combine this thermal conductivity equation with the basalt equation used in Sakatani et al. (2018) to account for the approximately 50/50 ratio between glass and minerals, leading to the final expression for JSC1A simulant particle with no microporosity, $k_{s i m}=1.62+7.61 e-3 * T$. Next, if we assume that the particles are somewhat porous, as described above, we must also attempt to account for the effects of microporosity on $\mathrm{k}_{\mathrm{sim}}$. Several empirical datasets exist in the literature to describe the effects of porosity on thermal conductivity (e.g., Woodside and Messmer, 1961b; Flynn et al., 2018). We ran a simple model of a block with randomly placed nonconnected spherical voids in order to determine the effects of vesicle-like porosity in a geologic material (Figure S2). The size of the voids was increased to increase porosity, with the simplification that radiative heat transfer in the voids is negligible. We found this simplification to be valid to within a few percent at the relatively low temperatures used in the Sakatani et al. (2018) measurements. The model resulted in the following correlation to adjust a material thermal conductivity value to account for the presence of vesicular microporosity: $k_{m}^{*}=k_{m} *\left(0.466 \phi^{2}-1.496 \phi+1.0\right)$. This equation is applied to $\mathrm{k}_{\text {sim }}$ when microporosity is included.

For the assumed emissivity, we use a value of 0.90 , rather than the original value of 1.0 used in Sakatani et al. (2018). Typical basalt emissivity spectra, such as from the ASU spectral library (Christensen et al., 2000) and the Salisbury and d'Aria (1992), show an integrated value of $\sim 0.95$. However, those values of $\sim 0.95$ are for directional emissivity, typically normal or nearnormal emission angle, whereas radiative heat transfer between surfaces is controlled by the hemispherical emissivity. Hemispherical emissivity tends to be smaller than directional emissivity due to a roll-off in emissivity at higher emission angles on most surfaces, except those that are extremely rough (perfectly Lambertian, Warren et al., 2019, Figure 16 within). With such a roll-off, the integral of the emission half-space is necessarily smaller than the normal emissivity. The ratio of hemispherical-to-normal emissivity for a non-metallic solid with a normal emissivity of 0.95 is approximately 0.94, according to (Touloukian and DeWitt, 1972). Thus, we adopt a nominal value for hemispherical emissivity of 0.90 . 
With all of the aforementioned revised assumptions, new values for $\mathrm{F}, \zeta$, and $\xi$ are calculated by fitting the model of Sakatani et al. $(2017 ; 2018)$ to the bulk thermal conductivity results for a given sample size fraction from Sakatani et al. (2018). $\zeta$ and $\xi$ are varied as free parameters to achieve the optimal least-squares fit to the temperature-dependent experimental data. This approach to constrain these parameters is possible due to the difference in temperature dependence between the radiative and solid conductivity terms, which are tuned by $\zeta$ and xi, respectively. The value of $\mathrm{F}$ is calculated from the best fit value of $\zeta$ using Equation 2 . The results are shown in Tables S4 and S5, in comparison to the original values.

The values of $\mathrm{F}$, which are not dependent on assumed porosity, have increased compared to the original values, but otherwise do not change with assumed porosity value. That is not to say that $\mathrm{F}$ is not a function of the porosity of a particulate assemblage; it certainly is. Rather, the value of $\mathrm{F}$ determined from experimental data is independent of the experimenter's knowledge of the sample porosity. The values of $\zeta$ and $\xi$, on the other hand, are affected by our knowledge (or assumptions) of sample porosity. With increasing assumed sample porosity, $\zeta$ must decrease to compensate and maintain the same values of $F$ and $k_{r}$. Conversely, $\xi$ must decrease with increasing porosity to compensate for a decrease in assumed particle coordination number so as to maintain the same value of $k_{s}$.

Finally, it should be noted that when these values of $\mathrm{F}$ are compared to the results of our numerical simulations, the difference in assumed emissivity must be accounted for. In our simulations, emissivity=1, whereas we now assume that the Sakatani et al. (2018) results are for particles with emissivity $=0.9$. Because $\mathrm{F}$ is a function of emissivity, we must adjust the experimental values of $\mathrm{F}$ in order to compare to our simulation results. We will assume that $\mathrm{F}$ is proportional to $\varepsilon /(2-\varepsilon)$, based on the parallel-plates heat transfer approximation (e.g., Sakatani et al., 2017) and thus calculated an adjusted value of $F^{*}=F *(2-\varepsilon) / \varepsilon$. The original values of $\mathrm{F}$ are shown in Tables $\mathrm{S} 4$ and $\mathrm{S} 5$, while the adjusted $F^{*}$ values are shown in later figures.

\section{Results}

The radiative exchange factor $(\mathrm{F})$ values determined from all simulations where nonisothermality is assumed to be negligible (i.e., where $k_{m}=30.0 \mathrm{~W} \mathrm{~m}^{-1} \mathrm{~K}^{-1}$ ) are shown in Figure 3 and summarized in Tables S1 and S2. Given that the random packings and ordered packings are systematically offset from each other, we fit separate empirical functions to them using Equation 4. The coefficients for the random packing fit line are $a=0.739, b=0.629$, and $c=1.031$. This trend line captures all random packing data points with an uncertainty of $\pm 10 \%$. Uncertainty should be increased to $\pm 25 \%$ for porosity values $>\sim 0.65$ in order to capture all data points, error bars, and regular packing data points (Figure S3). The regular packing data points are fit well with the following coefficients: $a=0.773, b=0.419$, and $c=1.180$. The fit is shown with the data on Figure 4 , along with the original and revised values for $\mathrm{F}$ from the experimental data of Sakatani et al. (2018) and unchanged experimental data for glass beads from Sakatani et al. (2017). The full results of the reevaluation of data from Sakatani et al. (2018) are provided in Tables S4 and S5.

Results for simulations where solid conductivity were varied to induce the nonisothermality effect are shown in Figure 5 and in Table S3. In this work, we parameterize results as a function of $(1-\phi) / \Lambda_{s}$ to incorporate the effects of porosity, whereas in previous works (Ryan et al., 2020; van Antwerpen et al., 2012; Singh and Kaviany, 1994), the non-isothermality factor was presented in terms of $\Lambda_{s}$ or $1 / \Lambda_{s}$. A fit to the new data uses the following function: 
$579 \quad($ Eq 12)

580

$$
f_{k}=a_{1} \tan ^{-1}\left(a_{2}\left(\frac{1-\phi}{\Lambda_{s}}\right)^{a_{3}}\right)+a_{4}
$$

$581 \quad$ where $\mathrm{a}_{1}=-0.500, \mathrm{a}_{2}=1.351, \mathrm{a}_{3}=0.741$, and $\mathrm{a}_{4}=1.007$.

582

Finally results of the simulations where spheres were roughened by the addition of spherical section craters on their surfaces are shown in Figure 6 and Table S6. The values for two simulations with roughened spheres are compared to the same packing geometries without

585 roughness added.

586

587

588

589

590

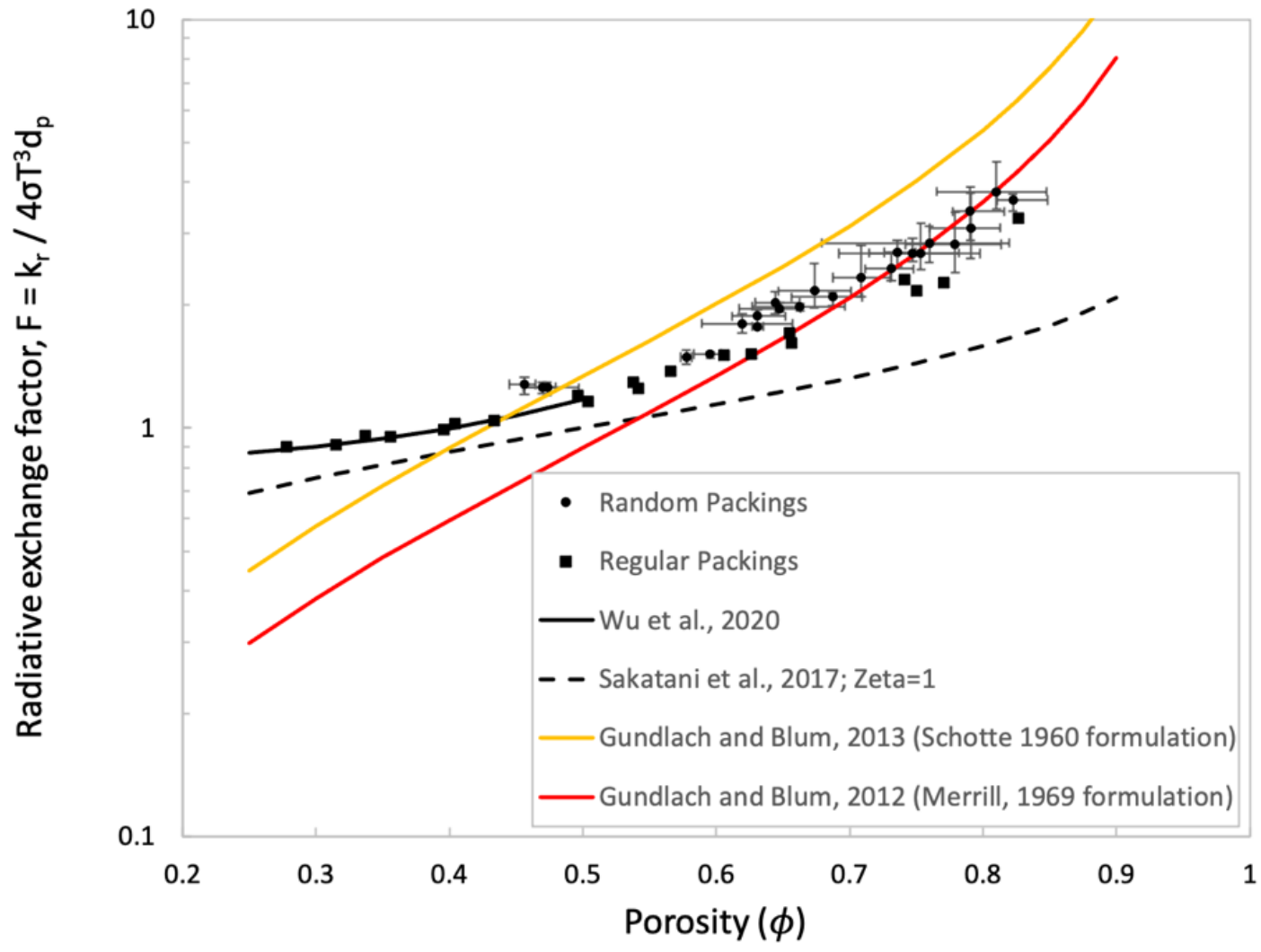

Figure 3. Numerical results for the radiative exchange factor as a function of porosity compared to models for regolith and sphere bed thermal conductivity. 
Manuscript submitted to JGR: Planets

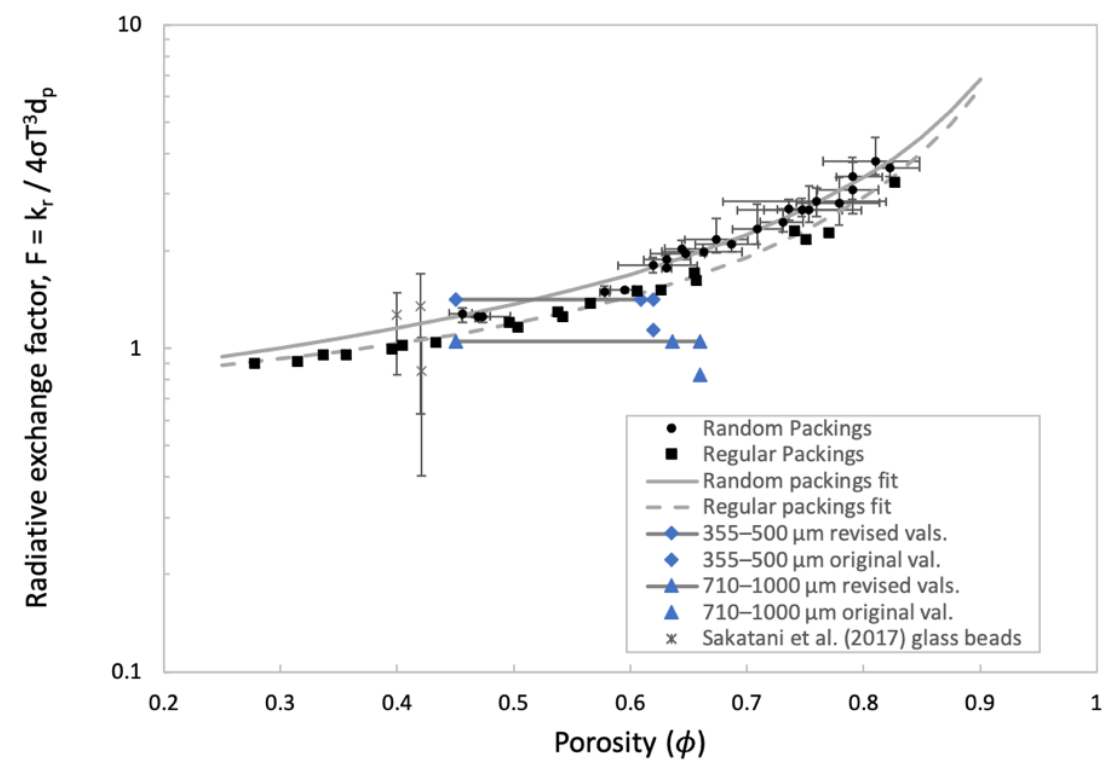

Figure 4. Numerical results for the radiative exchange factor as a function of porosity compared to experimental results from Sakatani et al. $(2017 ; 2018)$, adjusted to $\mathrm{F}^{*}$ to account for differences in sample emissivity $(\sim 0.9)$ compared to our simulations performed with unitary emissivity. Revised values of data collected for the JSC-1A lunar simulant are compared to completely unaltered values from Sakatani et al. (2018). For the reevaluated data, we show a line connecting three porosity points (from left to right): assumed lower end value of 0.45 , value water pycnometer measurements, and original value.

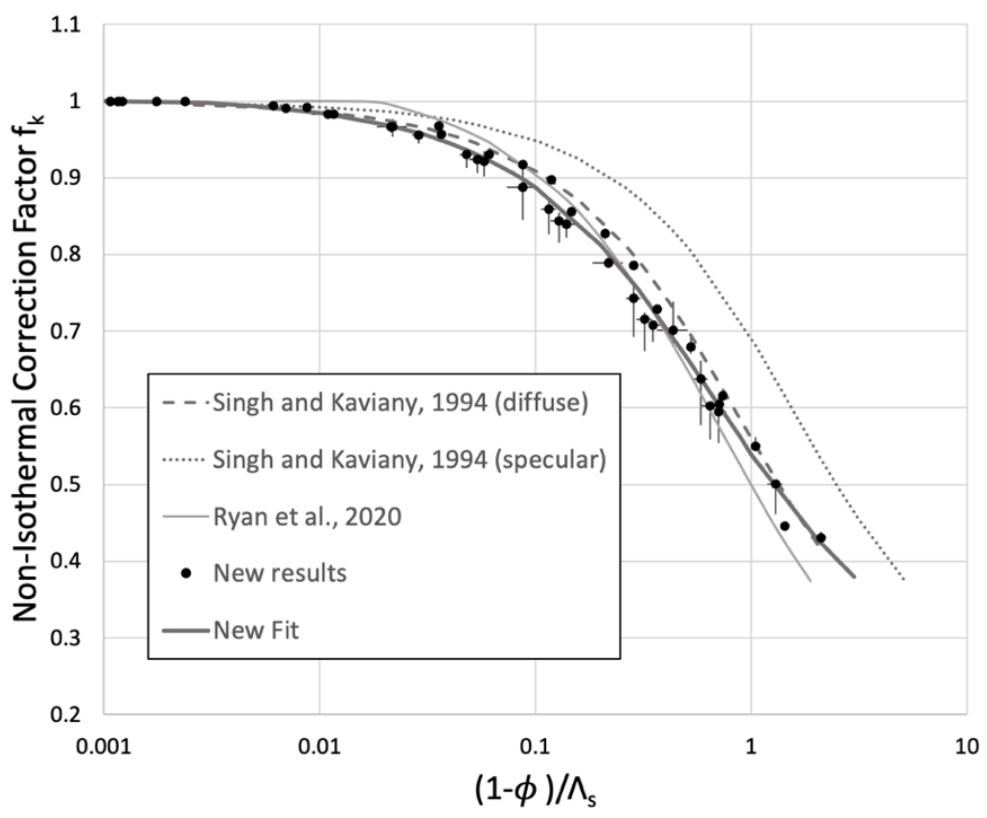

604 Figure 5. Results from simulations where particle non-isothermality was investigated. The new fit trendline is shown in addition to the trendlines from previous studies (Ryan et al., 2020; Singh 606 and Kaviany, 1994). 


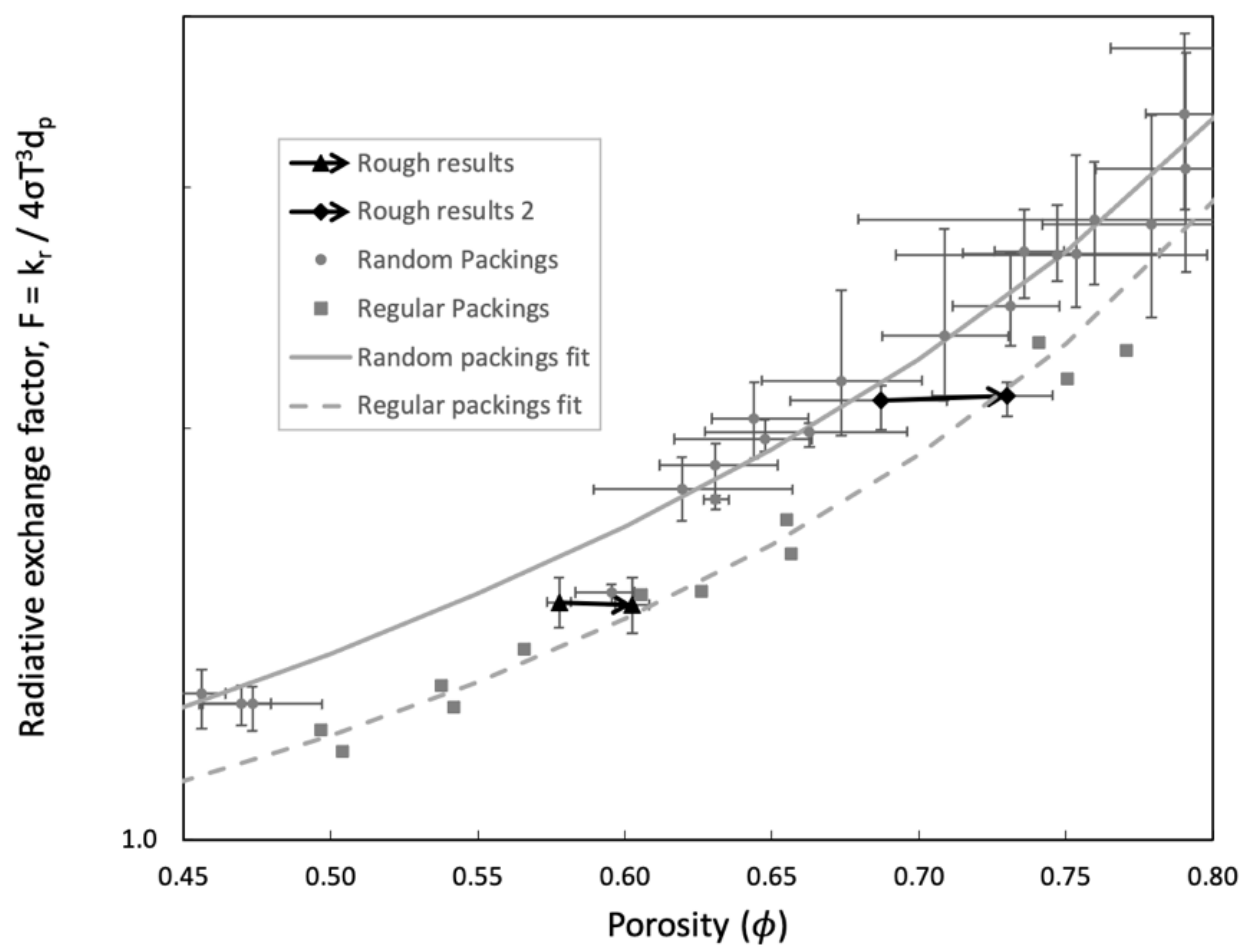

607

608

609

610

611

612

613

614

615

616

617

618

619

620

621

622

623

624

625

626

627

628

629

630

Figure 6. Results for two sets of simulations where the spheres were roughened by adding spherical section craters to the sphere surfaces, overlaid on a subset of full numerical results. Arrows point from original geometry without roughened spheres to results where spheres are roughened. Values provided in Table S6.

\section{Discussion}

The calculated values of the radiative exchange factor $F$ as a function of porosity for ordered packing are in very good agreement with the results by Wu et al. (2020), which were also determined numerically but with a matrix-based method. This gives us increased confidence that our numerical results are accurate to within a few percent. Our revised calculations of $F$ from the experimental data of Sakatani et al. (2018) are in relatively good agreement with our numerical results. Figure 4 shows revised values of $F$ and the range of potential porosity values for the two largest size fractions from their work. If we assume the most extreme scenario where both specimens have a true porosity of 0.45 , then the two values of $F$ bracket our results. This assumption would mean that the individual particles have high microporosity, with values of 0.30 for the $355-500 \mu \mathrm{m}$ sample and 0.39 for the $710-1000 \mu \mathrm{m}$ sample. These microporosity values are not unusual for basaltic cinders (e.g., Robertson and Peck, 1974).

The results of our simulations of roughened particles provides another mechanism aside from microporosity to increase the apparent bulk porosity of a specimen without necessarily increasing the value of $F$. In Figure 6, it is shown that the addition of craters to the surfaces of spheres, similar to open vesicles, does not increase the value of $F$ but does increase the measured porosity even though the packing density of the particles is unchanged. This behavior could partially explain why the experimental values of $F$, which were obtained with particles that are 
known to have rough, pitted surfaces, tend to be lower than our numerical results for perfect spheres. Finally, particle non-sphericity could also play a role (Garboczi, 2011). However, the effects of non-sphericity on radiative conductivity have yet to be studied thoroughly experimentally or numerically, though the model of Wood (2020) predicts that the radiative exchange factor is directly proportional to sphericity. Nonetheless, we find that the recalculated experimental values for $\mathrm{F}$ are in good agreement with our numerical results when considerations of microporosity and particle roughness are taken into account.

The values of $F$ in Figure 3 are consistently lower, by about $10-25 \%$, in regular packings than in random packings across the full porosity range investigated. We did not note any systematic differences within the random packings that could be attributed to packing style or whether or not the packings were physically realistic or not. We attribute the distinction between random and regular packings to a difference in the relative contributions of short-range and longrange radiative exchange (e.g., van Antwerpen et al., 2012). Ordered packings tend to have a higher number of spheres in their immediate proximity, compared to a random packing with a comparable porosity value. As such, a larger proportion of the view as seen from any surface location on a given sphere will be obscured by spheres in close relative proximity (i.e., immediate neighbors). It follows that the roll-off in view factor between any two given spheres in a regular packing would drop off sharply once the distance between the two spheres exceeds that of the immediately neighboring spheres. This would have the effect of increasing shortrange radiation and decreasing long-range radiation, compared to a random packing. We hypothesize that the decrease in long-range radiative heat transfer has a greater net effect than the increase in short-range radiative exchange, resulting in a net decrease in heat transfer in the ordered packings.

The overall trend between $\mathrm{F}$ and porosity cannot be well matched by the Sakatani et al. (2018) or the Gundlach and Blum $(2012 ; 2013)$ models across the full range of porosities tested. The Gundlach and Blum (2013) model matches well our results for random packings for porosities greater than $\gtrsim 0.70$. This is not surprising, given that their model expression was formulated using photon mean free path simulation data for porosity values exclusively in the range of 0.65-0.85 (Skorov et al., 2011).

In order to compare the three models, we calculated predicted effective particle sizes for the S-type asteroids (25143) Itokawa, (433) Eros, and (99942) Apophis and B-type asteroid (101955) Bennu (Table 1). As usual, these effective particle sizes are calculated assuming that the surface from which the thermal inertia value was derived is covered in a uniform blanket of particulate regolith. In the absence of rocks and boulders larger than the diurnal skin depth, the effective particle size is thought to reflect either the Sauter mean (Ryan et al., 2020) or the volumetric median particle diameter (Sakatani et al., 2018). The presence of boulders or exposed bedrock will typically shift the result towards a larger particle size, given that boulders typically have a higher thermal inertia than fine regolith. The magnitude of this shift will depend on the relative spatial abundance of boulders and on the difference in thermal inertia between the particulate regolith and the boulder components. On a planetary body like the Moon, this difference is very large (e.g., Bandfield et al., 2011). On Bennu, the difference can be very small or even non-existent (Rozitis et al., 2020; Cambioni et al., 2021).

To calculate the effective particle size for the S-type asteroids (Table 1), we use the same material properties and the same non-isothermal correction in all models so that the effect of the different $F$ versus phi model relationships across a range of thermal inertia values can be 
676

compared. For Bennu, we assume Cold Bokkeveld-like material properties (Opeil et al., 2020) and use a nominal thermal inertia value of $200 \mathrm{~J} \mathrm{~m}^{-2} \mathrm{~K}^{-1} \mathrm{~s}^{-1 / 2}$ and mean diurnal temperature of $260 \mathrm{~K}$ to represent the Hokioi Crater, the location of the Nightingale sample site (Rozitis et al., 2020). For Itokawa, we use a regolith-specific thermal inertia value of $203 \mathrm{~J} \mathrm{~m}^{-2} \mathrm{~K}^{-1} \mathrm{~s}^{-1 / 2}$ and global mean diurnal temperature of $300 \mathrm{~K}$ from Cambioni et al. (2019). We provide effective particle size estimates in Table 1 for regolith porosities in the range $0.40-0.90$ but otherwise do not perform a robust error analysis at this time, given that the aim of this exercise is to compare nominal model predictions.

Table 1. Example predicted effective particle diameters for four asteroids using different regolith thermal conductivity models assuming regolith porosity values in the range of 0.4-0.9. Predictions for Eros, Itokawa, and Apophis use S-type material properties from Gundlach and Blum (2013). Predictions for Bennu use material properties as described in Rozitis et al. (2020) with the exception of using Cold Bokkeveld thermal conductivity and heat capacity at $260 \mathrm{~K}$ from Opeil et al. 2020. The Sakatani model uses $\zeta=0.68+\left(7.6 * 10^{-5}\right) / D_{p}$ and $\xi=0.12$ (Wada et al., 2018). For our calculations, we use Equation 4 with the fit random packing fit parameters provided in the Results section to calculate radiative conductivity. To calculate solid conductivity, we use the Sakatani et al. (2017) expression (their Equation 19); using the Gundlach and Blum (2013) solid conductivity expression provides very similar results. Thermal inertia and mean temperature values for Eros come from Gundlach and Blum (2013) and references therein. Given that the thermal inertia of Apophis is not well constrained, we calculate particle sizes for the low, middle, and high best fit values from Licandro et al. (2016) with an average temperature of $250 \mathrm{~K}$ (Sorli and Hayne, 2020). TI is thermal inertia in units of $\mathrm{J} \mathrm{m}^{-2} \mathrm{~K}^{-1}$ $\mathrm{s}^{-1 / 2}$.

\begin{tabular}{lllllll}
\hline Model & $\begin{array}{l}\text { Eros } \\
\mathrm{TI}=150 \\
(\mathrm{~mm})\end{array}$ & $\begin{array}{l}\text { Bennu } \\
\text { Nightingale } \\
\mathrm{TI}=200 \\
(\mathrm{~mm})\end{array}$ & $\begin{array}{l}\text { Itokawa } \\
\text { Regolith } \\
\mathrm{TI}=200 \\
(\mathrm{~mm})\end{array}$ & $\begin{array}{l}\text { Apophis } \\
\mathrm{TI}=50 \\
(\mu \mathrm{m})\end{array}$ & $\begin{array}{l}\text { Apophis } \\
\mathrm{TI}=275 \\
(\mathrm{~cm})\end{array}$ & $\begin{array}{l}\text { Apophis } \\
\mathrm{TI}=500 \\
(\mathrm{~cm})\end{array}$ \\
\hline $\begin{array}{l}\text { This work } \\
\begin{array}{l}\text { Gundlach and } \\
\text { Blum (2013) }\end{array}\end{array}$ & $2.8-4.9$ & $8.0-8.2$ & $5.3-5.4$ & $450-560$ & $1.7-1.73$ & $5.7-5.9$ \\
$\begin{array}{l}\text { Sakatani et al. } \\
(2017)\end{array}$ & $9.3-24$ & $17.3-41.7$ & $3.0-6.7$ & $270-312$ & $1.0-2.2$ & $3.2-7.8$ \\
\hline
\end{tabular}

Our new model for random packings predicts particle sizes that fall within the range of predictions by the Gundlach and Blum (2013) model. The Gundlach and Blum model results are more sensitive to porosity than our model and lead to a wider range of predicted values, given the steeper slope in the relationship between F and porosity (Figure 3). The Sakatani model tends to predict much larger particle sizes, which is a direct result of its lower predicted values for $\mathrm{F}$, especially at higher porosities. To illustrate the relative relationships between porosity and thermal inertia, we plot the thermal inertia of $7.5 \mathrm{~mm}$ diameter regolith particles in Figure 7 using the model parameters used to calculate the Bennu particle sizes in Table 1. Although all three models predict an increase in thermal conductivity with increasing porosity, the magnitude 
714

\section{5}

of this increase relative to the accompanying decrease in regolith bulk density with increasing porosity causes the three models to behave very differently. The increase in conductivity with porosity in our model is approximately equivalent to the decrease in density. The Gundlach and Blum (2013) model outpaces the density decrease, whereas the Sakatani model falls behind it.

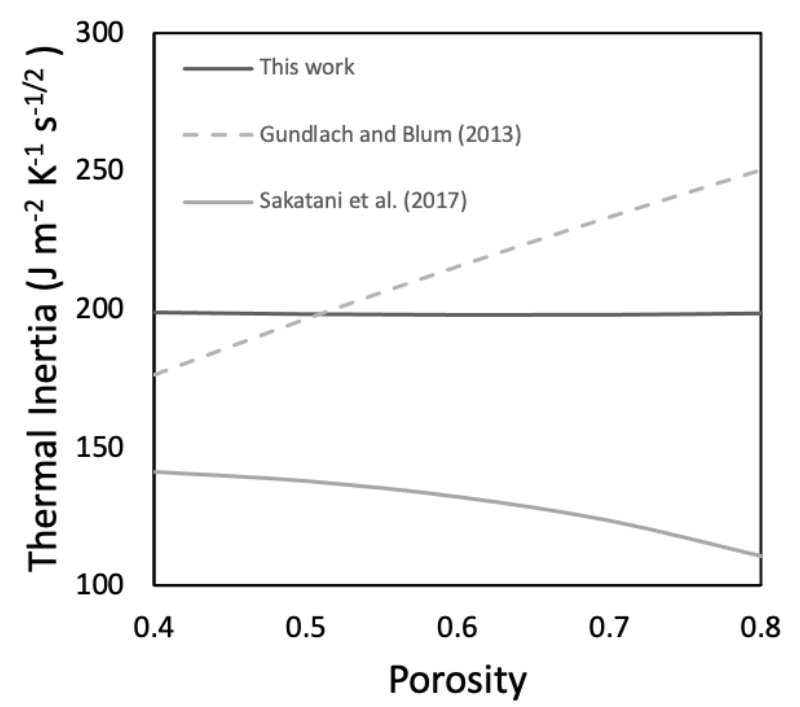

Figure 7. Calculated thermal inertia comparison for a hypothetical Bennu regolith particle of 7.5 mm diameter.

Finally, we have demonstrated that the non-isothermality effect in regoliths with different porosity values can be well-described by Equation 12 (Figure 5, "New Fit"). To visualize this result compared to the previous, porosity-insensitive results, we repeat the exercise from Ryan et al. (2020) and show the predicted particle size as a function of particle thermal conductivity for a regolith on Bennu with a thermal inertia of $200 \mathrm{~J} \mathrm{~m}^{-2} \mathrm{~K}^{-1} \mathrm{~s}^{-1 / 2}$, which is the upper end of the OSIRIS-REx Nightingale Sample site thermal inertia value calculated from Recon A mission phase data (Rozitis et al., 2020). The result is shown in Figure 8 overlain with thermal conductivity of the two Bennu boulder types, which may serve as the source material for the regolith particles at Nightingale. 


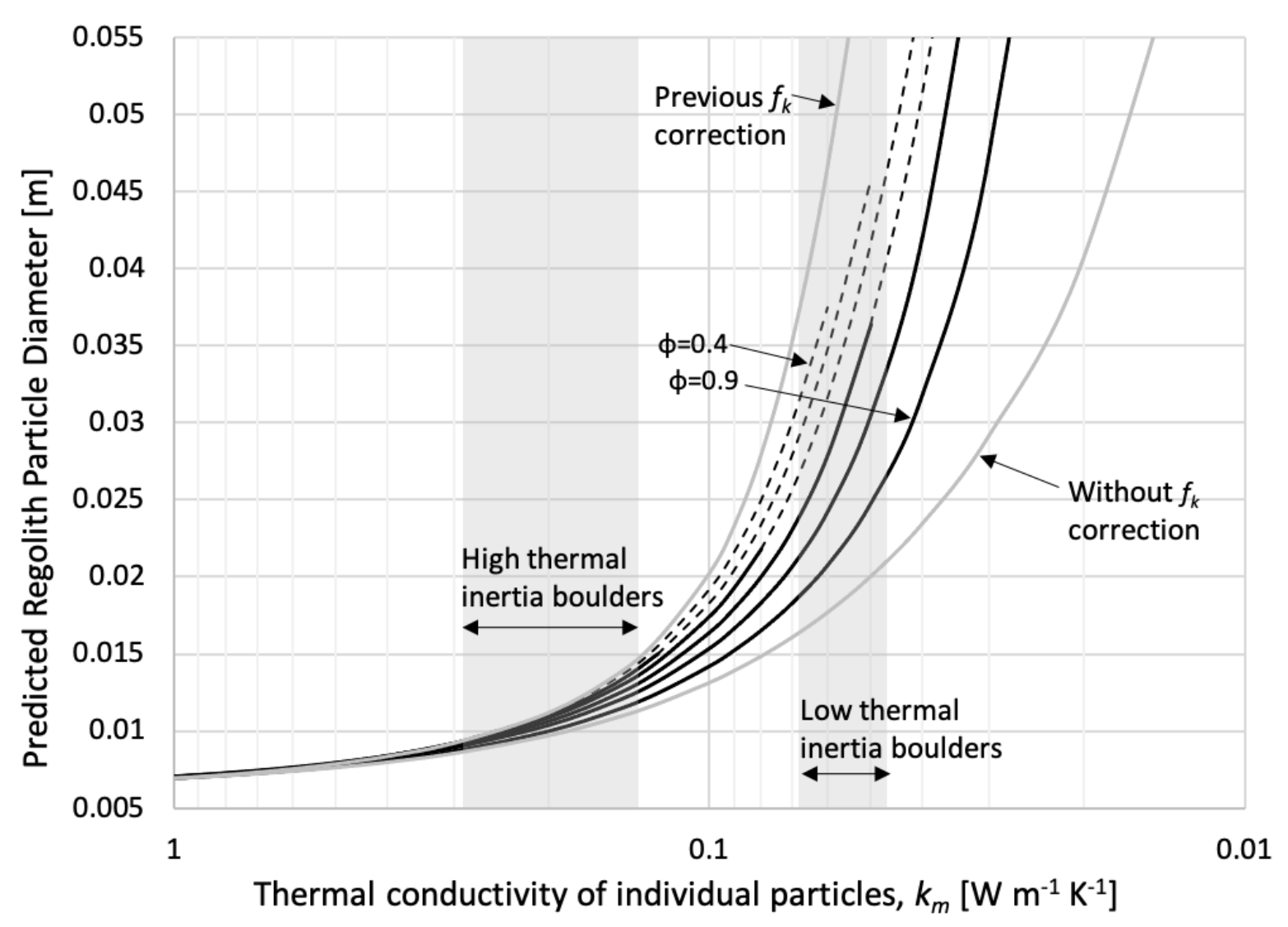

Figure 8. Example particle size prediction for the Nightingale sample site on Bennu (thermal inertia of $200 \mathrm{~J} \mathrm{~m}^{-2} \mathrm{~K}^{-1} \mathrm{~s}^{-1 / 2}$ at $265 \mathrm{~K}$ ) as a function of the conductivity of the material that makes up the regolith particles $\left(\mathrm{k}_{\mathrm{m}}\right)$. Predicted values using the new non-isothermality $\left(f_{k}\right)$ correlation the effects of porosity are shown as dark black solid and dashed lines. Lines change from solid to dashed when the predicted particle size exceeds the diurnal skin depth. Lines disappear completely when the prediction exceeds two diurnal skin depths. The lines are presented in order of assumed regolith porosity, with the two end values of 0.4 and 0.9 labeled. For comparison, the predictions are shown using the previous $f_{k}$ correction from Ryan et al. (2020) and without using any $f_{k}$ correction. The shape of those curves is insensitive to assumed regolith porosity; skin depth cutoffs however are sensitive to porosity but were not possible to clearly plot. All model parameters are the same as described in Ryan et al. (2020) and Rozitis et al. (2020), with the following exceptions: heat capacity is taken from the measurement of the Cold Bokkeveld meteorite by Opeil et al. (2020), particle density (or microporosity) varies with particle thermal conductivity using the model by Flynn et al. (2018), emissivity $=0.95$, and $\zeta=$ $0.68+\left(7.6 * 10^{-5}\right) / D_{p}$ (from Wada et al., 2018). Bennu low and high thermal inertia boulder values from Rozitis et al. (2020) were used to calculate the conductivity ranges, using the same assumptions for grain density and the relationship between porosity and microporosity described above. 
For a particulate regolith to have a lower thermal inertia compared to its source rock material, the size of the regolith particles should be smaller than one or a few skin depths (the exact cutoff is not yet known; Ryan et al., 2020). Otherwise, particulate regolith and rock should be indistinguishable. Several pieces of useful information may be extracted from Figure 8. First, if the regolith is sourced from the high thermal inertia boulders, the particle size prediction is well-constrained and is minimally sensitive to porosity. Conversely, if the regolith is sourced from the low thermal inertia boulders, the particle size is very poorly constrained without additional information, such as an estimate of porosity. For example, if we assume that the source rock material that composes the regolith at the Nightingale site has a thermal inertia of $200 \mathrm{~J} \mathrm{~m}^{-2} \mathrm{~K}^{-1} \mathrm{~s}^{-1 / 2}\left(k_{m} \approx 0.053 \mathrm{~W} \mathrm{~m}^{-1} \mathrm{~K}^{-1}\right)$, the particle size of the regolith could be anything larger than $\sim 2.25 \mathrm{~cm}$. However, if the images could for example be used to constrain that the effective regolith particle size to smaller than $3 \mathrm{~cm}$, then one could conclude that the regolith must have a porosity greater than $\sim 0.7$, if we maintain the assumption that the particles come from boulders with thermal inertia of $200 \mathrm{~J} \mathrm{~m}^{-2} \mathrm{~K}^{-1} \mathrm{~s}^{-1 / 2}$. One could also use a plot like this to estimate regolith particle thermal conductivity, with enough information. For example, if the effective particle size is known to be less than $1.8 \mathrm{~cm}$, we could conclude that the particles cannot be made of the low thermal inertia boulder material.

A recent study by Cambioni et al. (2021) presents evidence that the high thermal inertia boulders on Bennu and other primitive bodies are more likely to produce regolith particles than the low thermal inertia boulders due to different relative rates of fragmentation in response to meteoroid impacts and thermal fracturing. If the regolith particles at the Nightingale Site are indeed sourced predominantly from high thermal inertia boulders, then the effective particle size for this thermal inertia value would be $\sim 1-1.5 \mathrm{~cm}$ in diameter, which is consistent with observations of abundant resolved and unresolved particles $<2 \mathrm{~cm}$ (Burke et al., 2021; Walsh et al., in revision). However, the thermal inertia estimates for the Nightingale site still include contributions of rocks and fine regolith (Rozitis et al., 2020). Thus, we await thermal modeling results using the highest spatial resolution data from the TAG operation that might cover areas with only particles smaller than the diurnal skin depth before attempting a robust quantitative analysis of the thermophysical properties of the returned sample.

\section{Conclusions and Future Work}

We have numerically determined the effective radiative thermal conductivity and radiative exchange factor of random and regular packings of spheres in order to investigate the effects of porosity and particle thermal conductivity on the observed thermal inertia of airless body regolith. Our results are in agreement with experimental data from Sakatani et al. (2017; 2018) and show a new relationship between regolith radiative thermal conductivity, porosity, and the particle non-isothermality that was not predicted across the full range of porosity $(\sim 0.35-0.8)$ by any other models. We have also found that regular packings have a radiative exchange factor that is $10-25 \%$ lower than random packings in the range of porosities where both were examined $(\sim 0.45-0.80)$. As such, future investigators should not use regular packings as an approximation for random packings in studies of radiative heat transfer, despite their relative numerical and analytical convenience.

The resulting expression for the radiative thermal conductivity of regolith is represented in Equation 8, making use of our new expression and coefficients for the radiative exchange 
factor, $F$ (Equation $8, \mathrm{a}=0.739, \mathrm{~b}=0.629$, and $\mathrm{c}=1.031$ ), and the non-isothermality effect, $f_{k}$ (Equation 12, $\mathrm{a}_{1}=-0.500, \mathrm{a}_{2}=1.351, \mathrm{a}_{3}=0.741$, and $\mathrm{a}_{4}=1.007$ ).

There are several outstanding questions in the study of regolith thermal properties and the interpretation of thermal inertia results. We note a few high-priority items here that should be addressed in future studies:

- The effects of non-unitary emissivity on regolith bulk radiative conductivity should be incorporated into our porosity-dependent expressions for $F$ and $f_{k}$. Our model does not currently support non-unitary emissivity, but it is in development.

- The apparent particle-size dependence of experimental fit parameters $\zeta$ and $\xi$ (Sakatani et al., 2017; 2018) has yet to be conclusively explained. It is likely that more experimental data are needed to determine if this is a real phenomenon.

- The bulk radiative thermal conductivity of polydisperse particulates was shown to be represented by the Sauter mean particle diameter in our previous study (Ryan et al., 2020). However, we are concerned that the particle size ranges used in that work were too narrow to conclusively distinguish between the Sauter mean and the volumetric median (c.f., Sakatani et al., 2018) as the representative particle diameter. Furthermore, the solid conduction term may have a different representative particle size, given that the governing equations for heat flow through particle contacts differ significantly from those that describe radiative conduction. Detailed experimentation or very large numerical models will be required to capture the necessarily large representative volume elements with wide ranges of particle sizes. Numerical investigations might be better suited to a less-intensive discrete element method model where all particles are modeled as having an internally uniform temperature, but this would be at the cost of losing information on the non-isothermality effect.

- The bulk solid conduction of a particle assemblage is controlled by the details of the particle-to-particle contacts, which are affected by assemblage packing density and many properties of the individual particles, including shape, roughness, and surface energy (related to composition and surface cleanliness). Much progress has been made on this subject in recent years (e.g., Sakatani et al., 2018; Arakawa et al., 2019; Wood, 2020; Arakawa, 2020), but there are still uncertainties in how these effects might scale with particle size.

- The apparent thermal inertia of a surface will transition from being controlled by the properties of a particulate assemblage to the properties of a single particle (a.k.a. boulder) as the particle size exceeds the diurnal skin depth. The details of this transition are not known in detail, aside from the assumption that it will occur when the particle size approximately exceeds the diurnal skin depth. We intend to address this in our next manuscript.

- Finally, as mentioned in our discussion, it is unclear what temperature to use when interpreting model-derived thermal inertia values in terms of regolith particle size. Given the strongly temperature-dependent thermal conductivity of coarse regolith, the instantaneous thermal inertia will change throughout the diurnal cycle. However, we expect that the overall diurnal profile can be approximated with the thermal properties of the regolith at or near the mean diurnal temperature. This expectation should be verified under a range of conditions, including different regolith material properties, rotation periods, and heliocentric distances. 


\section{6}

857

858

859

860

861

862

863

864

865

866

867

868

869

870

871

872

873

874

875

876

877

878

\section{Acknowledgments and Data Availability}

This work was primarily funded by NASA Solar System Workings Grant 80NSSC21K0146. The authors acknowledge support from the Academies of Excellence on Complex Systems and Space, Environment, Risk and Resilience of the Initiative d'EXcellence (IDEX) Joint, Excellent, and Dynamic Initiative (JEDI) of the Université Côte d'Azur as well as from the Centre National d'Études Spatiales (CNES). B.R. acknowledges funding support from the UK Science and Technology Facilities Council (STFC). This material is based in part upon work supported by NASA under Contract NNM10AA11C issued through the New Frontiers Program. We thank Ron Ballouz for sharing his code for generating sphere packings using the method by Ringl et al. (2012). We also thank Mark Bentley for making his aggregate packing code publicly available on his GitHub page (https://github.com/msbentley/aggregate). The portion of the aggregate code that we modified to include periodicity is available in an external archive, along with our model geometry/solution files, script for processing model outputs, and a summary calculation spreadsheet (Ryan, 2022; https://doi.org/10.5281/zenodo.5839026). The authors declare no real or perceived conflicts of interest.

\section{References}

Arakawa, S., Tanaka, H., Katoaka, A., \& Nakamoto, T. (2017). Thermal conductivity of porous aggregates. Astronomy and Astrophysics, 608, L7. doi:10.1051/0004-6361/201732182

Arakawa, S., Tatsuuma, M., Sakatani, N., \& Nakamoto, T. (2019). Thermal conductivity and coordination number of compressed dust aggregates. Icarus, 324, 8-14. doi:10.1016/j.icarus.2019.01.022

Arakawa, S. (2020) Revisiting sticking property of submillimetre-sized aggregates. Monthly Notices of the Royal Astronomical Society, 496, 2786-2789. doi:10.1093/mnras/staa1764

Ballouz, R.-L., Walsh, K. J., Sánchez, P., Holsapple, K.A., Michel, P. Scheeres, D. J., et al., (2021). Modified granular impact force laws for the OSIRIS-REx touchdown on the surface of asteroid (101955) Bennu. Monthly Notices of the Royal Astronomical Society, 507, 50875105. doi: $10.1093 / \mathrm{mnras} / \mathrm{stab} 2365$

Bandfield, J.L., Ghent, R.R., Vasavada, A.R., Paige, D.A., Lawrence, S.J., \& Robinson, M.S. (2011) Lunar surface rock abundance and regolith fines temperatures derived from LRO Diviner Radiometer data. Journal of Geophysical Research, 116, E00H02. doi:10.1029/2011JE003866

Birch, F. \& Clark, H. (1940) The thermal conductivity of rocks and its dependence upon temperature and composition. American Journal of Science, 238(8), 529-558.

Birch, F. \& Law, R. R. (1935) Measurement of compressibility at high pressures and high temperatures. Bulletin of the Geological Society of America, 46, 1219-1250.

Breitbach, G., \& Barthels, H. (1980) The radiance heat transfer in the high temperature reactor core after failure of the afterheat removal systems. Nuclear Technology, 49(3), 392-399. doi:10.13182/NT80-A17687 
Burke, K. N., DellaGiustina, D. N., Bennett, C. A., Walsh, K. J., Pajola, M., Bierhaus, E. B., et al., (2021) Particle Size-Frequency Distributions of the OSIRIS-REx Candidate Sample Sites on Asteroid (101955) Bennu. Remote Sensing, 13, 1315. doi:10.3390/rs13071315

Calderón-Vásquez, I., Cortés, E., García, J., Segovia, V., Caroca, A., Sarmiento, C., et al. (2021) Review on modeling approaches for packed-bed thermal storage systems. Renewable and Sustainable Energy Reviews, 143, 110902. doi:10.1016/j.rser.2021.110902

Cambioni, S., Delbo, M., Ryan, A. J., Furfaro, R., \& Asphaug, E. (2019). Constraining the thermal properties of planetary surfaces using machine learning: Application to airless bodies. Icarus, 325, 16-30. doi:10.1016/j.icarus.2019.01.017

Cambioni, S., Delbo, M., Poggiali, G., Avdellidou, C., Ryan, A. J., et al. (2021) Fine-regolith production on asteroids controlled by rock porosity, Nature, 598, 49-52.

Choudhary, M. K. \& Potter, R. M. (2005) Heat Transfer in Glass-Forming Melts, in Properties of Glass-Formation Melts, edited by D. L. Pye, A. Montenaro, I. Joseph; CRC Press, Boca Raton.

Christensen, P. R., J. L. Bandfield, V. E. Hamilton, D. A. Howard, M. D. Lane, J. L. Piatek, S. W. Ruff, \& W. L. Stefanov (2000b), A thermal emission spectral library of rock-forming minerals, J. Geophys. Res., 105(E4), 9735-9739.

De Beer, M., Rousseau, P. G., \& du Toit, C. G. (2018). A review of methods to predict the effective thermal conductivity of packed pebble beds, with emphasis on the near-wall region. Nuclear Engineering and Design, 331, 248-262. doi:10.1016/j.nucengdes.2018.02.029

Delbo, M., M. Mueller, J. P. Emery, B. Rozitis, \& M. T. Capria (2015), Asteroid thermophysical modeling, in Asteroids IV, edited by P. Michel, F. DeMeo, \& W. F. Bottke, pp. 107-128, The University of Arizona Press, Tucson.

Emery, J. P., Fernández, Y. R., Kelley, M. S. P., nèe Crane, K. T. W., Hergenrother, C., Lauretta, D. S., et al. (2014). Thermal infrared observations and thermophysical characterization of OSIRIS-REx target asteroid (101955) Bennu. Icarus, 234(C), 17-35. doi:10.1016/j.icarus.2014.02.005

Fergason, R. L., P. R. Christensen, J. F. Bell III, M. P. Golombek, K. E. Herkenhoff, \& Kieffer, H. H. (2006), Physical properties of the Mars Exploration Rover landing sites as inferred from Mini-TES-derived thermal inertia, Journal of Geophysical Research, 111(E2), E02S21. doi:10.1029/2005JE002583

Flynn, G. J., Consolmagno, G. J., Brown, P., \& Macke, R. J. (2018). Physical properties of the stone meteorites: Implications for the properties of their parent bodies. Chemie de Erde, 78, 269-298. doi:10.1016/j.chemer.2017.04.002

Garboczi, E. J. (2011) Three dimensional shape analysis of JSC-1A simulated lunar regolith particles. Powder Technology, 207, 96-103. doi:10.1016/j.powtec.2010.10.014

Grott, M., Knollenberg, J., Hamm, M., Ogawa, K., Jaumann, R., Otto, K., et al. (2019). Low thermal conductivity boulder with high porosity identified on C-type asteroid (162173) Ryugu. Nature Astronomy, 3, 971-976. doi:10.1038/s41550-019-0832-x 
918

Gundlach, B., \& Blum, J. (2012). Outgassing of icy bodies in the Solar System - II: Heat transport in dry, porous surface dust layers. Icarus, 219(2), 618-629. doi:10.1016/j.icarus.2012.03.013

Gundlach, B., \& Blum, J. (2013). A new method to determine the grain size of planetary regolith. Icarus, 223(1), 479-492. doi:10.1016/j.icarus.2012.11.039

Henderson, B. G. \& Jakosky, B. M. (1994) Near-surface thermal gradients and their effects on mid-infrared emission spectra of planetary surfaces. Journal of Geophysical Research, 99(E9), 19063-19073.

Hitti, K., \& Bernacki, M. (2013). Optimized Dropping and Rolling (ODR) method for packing of poly-disperse spheres. Applied Mathematical Modelling, 37(8), 5715-5722, doi:10.1016/j.apm.2012.11.018

Jakob, M. (1949), Heat Transfer, 1st ed., John Wiley \& Sons, New York.

Kaviany, M. (1995), Principles of Heat Transfer in Porous Media, 2nd ed., Springer, New York.

Licandro, J., Müller, T., Alvarez, C., Alí-Lagoa, V., \& Delbo, M. (2016) GTC/CanariCam observations of (99942) Apophis. Astronomy \& Astrophysics, 585, A10. doi: 10.1051/0004$6361 / 201526888$

McKay, D. S., Carter, J. L., Boles, W. W., Carlton, C. A., \& Allton, J. H. (1994) JSC-1: A new lunar soil stimulant. Proc., Symp. 94, ASCE, New York, 857-866.

Merrill, R. B. (1969). Thermal conduction through an evacuated idealized powder over the temperature range of 100 to $500 \mathrm{~K}$, NASA Technical Note D-5063. https://ntrs.nasa.gov/search.jsp?R=19690010650.

Murdoch, N., Sánchez, P., Schwartz, S. R., \& Miyamoto, H. (2015) Asteroid Surface Geophysics, in Asteroids IV, edited by P. Michel, F. DeMeo, \& W. F. Bottke, pp. 767-793, The University of Arizona Press, Tucson.

Nakamura, T., Noguchi, T., Tanaka, M., Zolensky, M.E., Kimura, M., Tsuchiyama, A., et al. (2011). Itokawa Dust Particles: A Direct Link Between S-Type Asteroids and Ordinary Chondrites. Science, 333(6046), 1113-1116. doi: 10.1126/science.1207758

Okada, T., Yamamoto, Y., Inoue, T., Shirai, K., Arai, T., Ogawa, K., et al. (2006). Thermal radiometry of asteroid Itokawa by the XRS onboard Hayabusa. Lunar and Planetary Science XXXVII, Abstract 1965.

Opeil, C. P., Britt, D. T., Macke, R. J., \& Consolmagno, G. J. (2020) The surprising thermal properties of CM carbonaceous chondrites. Meteoritics \& Planetary Science. doi: 10.1111/maps.13556

Presley, M. A., \& Christensen, P. R. (1997). Thermal conductivity measurements of particulate materials: 1. A review. Journal of Geophysical Research, 102(E3), 6535-6549. doi:10.1029/96JE03302

Ringl, C., Bringa, E. M., Bertoldi, D. S., \& Urbassek, H. M. (2012) collisions of porous clusters: A granular-mechanics study of compaction and fragmentation. The Astrophysical Journal, 752(151). doi:10.1088/0004-637X/752/2/151 
Robertson, E. C., \& Peck, D. L. (1974), Thermal Conductivity of Vesicular Basalt from Hawaii. Journal of Geophysical Research, 79(32), 4875-4888.

Robold, K. (1982), Wärmetransport im inneren und in der Randzone von Kugeischüttungen, Dissertation Jul-1796, Nuclear Research Center, Jülich.

Rozitis B., Ryan, A. J., Emery, J. P., Christensen, P. R., Hamilton, V. E., Simon, A. A., et al. (2020) Thermal Inertia Maps of (101955) Bennu from OSIRIS-REx Infrared Observations. Science Advances, 6, eabc3699. doi: 10.1126/sciadv.abc3699

Rozitis, B. \& Green, S. F. (2011) Directional characteristics of thermal-infrared beaming from atmosphereless planetary surfaces - a new thermophysical model. Monthly Notices of the Royal Astronomical Society, 415, 2042-2062.

Ryan, A. J. (2022). Data and scripts for manuscript "Full-field modeling of heat transfer in asteroid regolith 2: Effects of porosity" [Data set]. Zenodo. doi:10.5281/zenodo.5839026

Ryan, A. J., Pino Muñoz, D., Bernacki, M., \& Delbo, M. (2020) Full-Field Modeling of Heat Transfer in Asteroid Regolith: Radiative Thermal Conductivity of Polydisperse Particulates. Journal of Geophysical Research: Planets, 125, e2019JE006100. doi:10.1029/2019JE006100

Sakatani, N., Ogawa, K., Arakawa, M., \& Tanaka, S. (2018). Thermal conductivity of lunar regolith simulant JSC-1A under vacuum. Icarus, 309, 13-24. doi:10.1016/j.icarus.2018.02.027

Sakatani, N., Ogawa, K., Iijima, Y., Arakawa, M., Honda, R., \& Tanaka, S. (2017). Thermal conductivity model for powdered materials under vacuum based on experimental studies. AIP Advances, 7(1), 015310-24. doi:10.1063/1.4975153

Salisbury, J. W. \& D'Aria, D. M. (1992) Emissivity of terrestrial materials in the 8-14 micrometer atmospheric window. Remote Sensing of Environment, 42, 83-106.

Schotte, W. (1960). Thermal conductivity of packed beds. AIChE Journal, 6(1), 63-67. doi:10.1002/aic.690060113.

Shimaki, Y., Senshu, H., Sakatani, N., Okada, T., Fukuhara, T., Tanaka, S., et al. (2020) Thermophysical properties of the surface of asteroid 162173 Ryugu: Infrared observations and thermal inertia mapping. Icarus, 348, 113835. doi:10.1016/j.icarus.2020.113835

Sibille, L., Carpenter, P., Schlagheck, R., \& French, R. A. (2006) Lunar Regolith Simulant Materials: Recommendations for Standardization, Production, and Usage. NASA Technical Report 2006-214605.

Singh, B. P., \& Kaviany, M. (1994). Effect of solid conductivity on radiative heat transfer in packed beds. International Journal of Heat and Mass Transfer, 37(16), 2579-2583, doi:10.1016/0017-9310(94)90295-X

Skorov, Y., van Lieshout, R., Blum, J., \& Keller, H. U. (2011) Activity of comets: Gas transport in the near-surface porous layers of a cometary nucleus. Icarus, 212, 867-876. doi:10.1016/j.icarus.2011.01.018

Sorli, K. C. \& Hayne, P. O. (2020) Thermophysical modeling of 99942 Apophis: Estimations of surface temperature during the April 2029 close approach. Apophis T-9 Years 2020, LPI Contrib. No. 2242. Abstract 2069. 
1000

1001

1002

1003

1004

1005

1006

1007

1008

1009

1010

1011

1012

1013

1014

1015

1016

1017

1018

1019

1020

1021

1022

1023

1024

1025

1026

1027

1028

1029

1030

1031

1032

1033

1034

1035

Tamari, S., Samaniego- Martínez, D., Bonola, I., Banbala, E. R., Ordaz-Chaparro, V. (2005) Particle Density of Volcanic Scoria Determined by Water Pycnometry. Geotechnical Testing Journal, 28(4), GTJ12675.

Tausendschön, J. \& Radl, S. (2021) Deep neural network-based heat radiation modelling between particles and between walls and particles. International Journal of Heat and Mass Transfer, 177, 121557. doi:10.1016/j.ijheatmasstransfer.2021.121557

Taylor, L. A., Hill, E., Liu, Y., \& Day, J. M. (2005). JSC-1 as the Lunar Soil Simulant of Choice. Meteoritics and Planetary Science Supplement, 40, 5180.

Touloukian, Y. S. \& DeWitt, D. P. (1972) Thermophysical Properties of Matter - The TPRC Data Series. Volume 8. Thermal Radiative Properties - Nonmetallic Solids.

van Antwerpen, W., Rousseau, P. G., \& du Toit, C. G. (2012). Multi-sphere Unit Cell model to calculate the effective thermal conductivity in packed pebble beds of mono-sized spheres. Nuclear Engineering and Design, 247, 183-201. doi:10.1016/j.nucengdes.2012.03.012

van Antwerpen, W., du Toit, C. G., \& Rousseau, P. G. (2010). A review of correlations to model the packing structure and effective thermal conductivity of packed beds of mono-sized spherical particles. Nuclear Engineering and Design, 240(7), 1803-1818. doi:10.1016/j.nucengdes.2010.03.009

Vortmeyer, D. (1979) Wärmestrahlung in dispersen Feststoffsystemen. Chem. Ing. Tech., 51(9), 839-851.

Wada, K., Grott, M., Michel, P., Walsh, K. J., Barucci, A. M., Biele, J., et al. (2018) Asteroid Ryugu before the Hayabusa2 encounter. Progress in Earth and Planetary Science, 5(82). doi:10.1186/s40645-018-0237-y

Walsh, K. J., Bierhaus, E.B., Lauretta, D. S., Nolan, M.C., Ballouz, R.-L., Bennet, C. A., et al. (in revision). Assessing the sampleability of Bennu's surface for the OSIRIS-REx asteroid sample return mission. Space Science Reviews.

Watson, K. (1964). The thermal conductivity measurements of selected silicate powders in vacuum from 150 degrees-350 degrees $K$ (Doctoral dissertation). Pasadena, CA: California Institute of Technology. https://resolver.caltech.edu/CaltechETD:etd-11042002-153232.

Warren, T. J., Bowles, N. E., Donaldson Hanna, K., \& Bandfield, J. L. (2019). Modeling the angular dependence of emissivity of randomly rough surfaces. Journal of Geophysical Research: Planets, 124, 585-601. doi:10.1029/2018JE005840

Wechsler, A. E., Glaser, P. E., \& Fountain, J. A. (1972). Thermal Properties of Granulated Materials. In J. W. Lucas (Ed.), Thermal Characteristics of the Moon. Cambridge, MA: MIT Press.

Wesselink, A. J. (1948). Heat conductivity and nature of the lunar surface material. Bulletin of the Astronomical Institutes of the Netherlands, 10(390), 351-363.

Wood, S. E. (2020) A mechanistic model for the thermal conductivity of planetary regolith: 1 . The effects of particle shape, composition, cohesion, and compression at depth, Icarus, 352, 113964. doi:10.1016/j.icarus.2020.113964 
1036 Woodside, W. \& Messmer, J. H. (1961). Thermal conductivity in porous media II. Consolidated 1037 rocks. Journal of Applied Physics, 32, 1699-1706. doi:10.1063/1.1728420

1038 Wu, H., Gui, H., Yang, X., Tu, J., \& Jiang, S. (2020). A matrix model of particle-scale radiative 1039

1040 heat transfer in structured and randomly packed pebble bed. International Journal of Thermal Sciences, 153, 106334.

1041 Zeng, X., He, C., Oravec, H., Wilkinson, A., Agui, J., \& Asnani, V. (2010). Geotechnical properties of JSC-1A lunar soil simulant. Journal of Aerospace Engineering, 23(2), 111-116. doi:10.1061/(ASCE)AS.1943-5525.0000014 